\title{
Supplementary Material: A Lagrangian Analysis of the Dynamical and Thermodynamic Drivers of Greenland Melt Events during 1979-2017
}

Mauro Hermann ${ }^{1}$, Lukas Papritz ${ }^{1}$, and Heini Wernli ${ }^{1}$

${ }^{1}$ Institute for Atmospheric and Climate Science, ETH Zürich, Zurich, Switzerland

Correspondence: Mauro Hermann (mauro.hermann@env.ethz.ch) 
Table S1. Melt events EV1-31 listed with their start and end date ("YYYY-MM-DD HH"), duration (dur.), maximum elevation (ME), T2M at maximum elevation $\left(T 2 M_{a v g}^{M E}\right)$, minimum melt extent (min. A.) and maximum melt extent (max. A.) - sorted by date.

\begin{tabular}{cccccccc} 
ID & start & end & $\begin{array}{c}\text { dur. } \\
\text { [d] }\end{array}$ & $\begin{array}{c}\text { ME } \\
\text { [m] }\end{array}$ & $\begin{array}{c}\text { T2M avg } \\
\text { [ }{ }^{\circ} \mathbf{C} \text { ] }\end{array}$ & $\begin{array}{c}\text { min. A. } \\
\text { [frac] }\end{array}$ & $\begin{array}{c}\text { max. A. } \\
\text { [frac] }\end{array}$ \\
\hline 1 & $1979-07-2418$ & $1979-07-2618$ & 2.25 & 2606 & -1.17 & 0.32 & 0.70 \\
2 & $1981-06-2518$ & $1981-06-2718$ & 2.25 & 2549 & -1.29 & 0.30 & 0.62 \\
3 & $1981-07-2112$ & $1981-07-2418$ & 3.5 & 2729 & 2.44 & 0.25 & 0.59 \\
4 & $1983-06-3018$ & $1983-07-0118$ & 1.25 & 2606 & -0.27 & 0.25 & 0.67 \\
5 & $1984-07-0318$ & $1984-07-0818$ & 5.25 & 2549 & -1.72 & 0.19 & 0.68 \\
6 & $1984-07-2218$ & $1984-07-2418$ & 2.25 & 2537 & -0.57 & 0.22 & 0.66 \\
7 & $1984-07-2618$ & $1984-07-2718$ & 1.25 & 2333 & -0.06 & 0.34 & 0.61 \\
8 & $1985-08-1218$ & $1985-08-1418$ & 2.25 & 2810 & -0.51 & 0.23 & 0.54 \\
9 & $1986-07-0218$ & $1986-07-0318$ & 1.25 & 2394 & 0.67 & 0.16 & 0.54 \\
10 & $1986-07-2018$ & $1986-07-2218$ & 2.25 & 2729 & 2.43 & 0.29 & 0.53 \\
11 & $1987-06-1218$ & $1987-06-1318$ & 1.25 & 2717 & -1.17 & 0.26 & 0.56 \\
12 & $1987-06-1818$ & $1987-06-2218$ & 4.25 & 2826 & -1.87 & 0.13 & 0.63 \\
13 & $1987-08-0718$ & $1987-08-1018$ & 3.25 & 2734 & -1.07 & 0.15 & 0.72 \\
14 & $1988-07-0412$ & $1988-07-1218$ & 8.5 & 2658 & -0.10 & 0.20 & 0.69 \\
15 & $1988-07-2118$ & $1988-07-2218$ & 1.25 & 2413 & 0.27 & 0.22 & 0.63 \\
16 & $1989-07-1318$ & $1989-07-2018$ & 7.25 & 2810 & -1.27 & 0.24 & 0.69 \\
17 & $1990-07-2818$ & $1990-07-3018$ & 2.25 & 2748 & -0.43 & 0.18 & 0.70 \\
18 & $1990-08-0518$ & $1990-08-0618$ & 1.25 & 2628 & -1.27 & 0.24 & 0.63 \\
19 & $1991-06-1818$ & $1991-06-1918$ & 1.25 & 2810 & -1.62 & 0.19 & 0.57 \\
20 & $1991-06-2618$ & $1991-06-2718$ & 1.25 & 2394 & -1.53 & 0.25 & 0.52 \\
21 & $1991-06-2912$ & $1991-07-0218$ & 3.5 & 2606 & -0.47 & 0.17 & 0.53 \\
22 & $1991-07-0418$ & $1991-07-1318$ & 9.25 & 3000 & -1.46 & 0.14 & 0.68 \\
23 & $1994-07-0718$ & $1994-07-0818$ & 1.25 & 2443 & 0.55 & 0.27 & 0.68 \\
24 & $1995-06-3018$ & $1995-07-0318$ & 3.25 & 2810 & -0.25 & 0.17 & 0.65 \\
25 & $1995-07-1218$ & $1995-07-1618$ & 4.25 & 2969 & -0.36 & 0.24 & 0.77 \\
26 & $1997-08-1118$ & $1997-08-1318$ & 2.25 & 2970 & 0.99 & 0.23 & 0.66 \\
27 & $1998-08-0118$ & $1998-08-0318$ & 2.25 & 2637 & -0.83 & 0.22 & 0.61 \\
28 & $1999-06-2818$ & $1999-07-0318$ & 5.25 & 2721 & -0.62 & 0.17 & 0.70 \\
29 & $1999-07-2518$ & $1999-07-3118$ & 6.25 & 2628 & -0.89 & 0.20 & 0.64 \\
11 & $2000-07-2818$ & $2000-07-2918$ & 1.25 & 2637 & 0.49 & 0.39 & 0.70
\end{tabular}


Table S2. As Table S1 for EV32-64.

\begin{tabular}{|c|c|c|c|c|c|c|c|}
\hline ID & start & end & $\begin{array}{l}\text { dur. } \\
\text { [d] }\end{array}$ & $\begin{array}{l}\text { ME } \\
{[\mathrm{m}]}\end{array}$ & $\begin{array}{c}\text { T2 } \mathbf{M}_{\text {avg }}^{\mathrm{ME}} \\
{\left[{ }^{\circ} \mathrm{C}\right]}\end{array}$ & $\begin{array}{c}\text { min. A. } \\
\text { [frac] }\end{array}$ & $\begin{array}{c}\max . \text { A. } \\
\text { [frac] }\end{array}$ \\
\hline 32 & 2000-08-01 00 & 2000-08-02 18 & 2 & 2729 & 0.61 & 0.34 & 0.59 \\
\hline 33 & 2000-08-19 18 & 2000-08-21 18 & 2.25 & 2634 & 0.43 & 0.19 & 0.53 \\
\hline 34 & 2002-06-11 18 & 2002-06-14 18 & 3.25 & 2810 & -0.67 & 0.13 & 0.71 \\
\hline 35 & $2002-06-2718$ & 2002-07-03 18 & 6.25 & 3156 & -0.44 & 0.25 & 1.08 \\
\hline 36 & 2002-07-06 18 & 2002-07-07 18 & 1.25 & 2444 & 1.23 & 0.18 & 0.64 \\
\hline 37 & 2002-07-20 18 & 2002-07-21 18 & 1.25 & 2719 & -0.67 & 0.28 & 0.70 \\
\hline 38 & 2003-08-26 18 & 2003-08-30 18 & 4.25 & 2729 & -0.30 & 0.15 & 0.63 \\
\hline 39 & 2004-06-18 12 & 2004-06-23 18 & 5.5 & 2826 & -1.05 & 0.15 & 0.62 \\
\hline 40 & 2004-07-05 18 & 2004-07-12 18 & 7.25 & 3175 & -0.15 & 0.23 & 0.86 \\
\hline 41 & 2004-08-11 18 & 2004-08-12 18 & 1.25 & 2810 & -0.06 & 0.36 & 0.69 \\
\hline 42 & 2005-06-13 18 & 2005-06-16 18 & 3.25 & 2628 & 0.67 & 0.17 & 0.70 \\
\hline 43 & 2005-07-02 18 & 2005-07-03 18 & 1.25 & 2486 & -0.17 & 0.23 & 0.68 \\
\hline 44 & 2005-07-13 18 & 2005-07-15 18 & 2.25 & 2729 & 2.98 & 0.35 & 0.66 \\
\hline 45 & $2005-07-2118$ & 2005-07-30 18 & 9.25 & 2916 & -0.58 & 0.28 & 0.91 \\
\hline 46 & 2006-07-19 18 & 2006-07-28 18 & 9.25 & 3100 & -0.51 & 0.22 & 0.85 \\
\hline 47 & 2006-08-03 18 & 2006-08-05 18 & 2.25 & 2606 & -0.98 & 0.26 & 0.65 \\
\hline 48 & $2006-08-1500$ & 2006-08-18 18 & 4 & 2758 & -0.91 & 0.29 & 0.78 \\
\hline 49 & 2007-06-10 18 & 2007-06-13 18 & 3.25 & 2637 & 1.88 & 0.20 & 0.63 \\
\hline 50 & 2007-06-22 18 & 2007-06-29 18 & 7.25 & 2637 & 5.04 & 0.18 & 0.73 \\
\hline 51 & 2007-07-06 18 & 2007-07-22 18 & 16.25 & 2658 & 0.70 & 0.20 & 0.74 \\
\hline 52 & 2007-07-24 18 & $2007-07-2518$ & 1.25 & 2381 & -2.37 & 0.27 & 0.65 \\
\hline 53 & 2008-06-13 18 & 2008-06-14 18 & 1.25 & 2637 & 1.14 & 0.39 & 0.67 \\
\hline 54 & 2008-06-17 18 & 2008-06-18 18 & 1.25 & 2482 & 2.37 & 0.18 & 0.55 \\
\hline 55 & 2008-07-05 18 & 2008-07-06 18 & 1.25 & 2786 & -0.91 & 0.40 & 0.76 \\
\hline 56 & 2008-07-28 18 & 2008-07-31 18 & 3.25 & 2537 & 0.59 & 0.19 & 0.73 \\
\hline 57 & 2009-07-06 18 & 2009-07-16 18 & 10.25 & 2943 & -0.10 & 0.25 & 0.82 \\
\hline 58 & 2009-07-20 18 & 2009-07-23 18 & 3.25 & 2486 & 0.09 & 0.22 & 0.71 \\
\hline 59 & 2010-07-18 18 & 2010-07-19 18 & 1.25 & 2486 & 0.11 & 0.13 & 0.60 \\
\hline 60 & $2010-07-2318$ & $2010-07-2718$ & 4.25 & 2486 & -0.88 & 0.20 & 0.63 \\
\hline 61 & 2010-07-29 18 & 2010-08-02 18 & 4.25 & 2537 & -1.22 & 0.20 & 0.64 \\
\hline 62 & 2010-08-09 18 & 2010-08-10 18 & 1.25 & 2455 & -0.28 & 0.14 & 0.49 \\
\hline 63 & 2010-08-15 18 & 2010-08-16 18 & 1.25 & 2628 & -0.26 & 0.16 & 0.50 \\
\hline 64 & 2011-06-13 18 & 2011-06-15 18 & 2.25 & 2856 & 0.93 & 0.10 & 0.68 \\
\hline
\end{tabular}


Table S3. As Table S1 for EV65-77.

\begin{tabular}{|c|c|c|c|c|c|c|c|}
\hline ID & start & end & $\begin{array}{c}\text { dur. } \\
\text { [d] }\end{array}$ & $\begin{array}{l}\text { ME } \\
{[\mathrm{m}]}\end{array}$ & $\begin{array}{c}\mathbf{T} \mathbf{M}_{\mathrm{avg}}^{\mathrm{ME}} \\
{\left[{ }^{\circ} \mathbf{C}\right]}\end{array}$ & $\begin{array}{c}\min . A . \\
\text { [frac] }\end{array}$ & $\begin{array}{c}\max . A . \\
{[\text { frac] }}\end{array}$ \\
\hline 65 & 2011-07-06 18 & 2011-07-10 18 & 4.25 & 2916 & -0.78 & 0.24 & 0.73 \\
\hline 66 & 2011-07-13 18 & $2011-07-1518$ & 2.25 & 2455 & 0.20 & 0.25 & 0.76 \\
\hline 67 & 2011-07-18 18 & $2011-07-2318$ & 5.25 & 2526 & 0.03 & 0.21 & 0.69 \\
\hline 68 & 2012-06-15 18 & 2012-06-29 18 & 14.25 & 2916 & -1.81 & 0.18 & 0.81 \\
\hline 69 & 2012-07-02 18 & 2012-07-17 18 & 15.25 & 3175 & -0.13 & 0.23 & 1.21 \\
\hline 70 & 2012-07-27 18 & 2012-08-08 18 & 12.25 & 3100 & -1.08 & 0.22 & 0.96 \\
\hline 71 & 2013-07-24 18 & 2013-07-28 18 & 4.25 & 2826 & -2.44 & 0.17 & 0.71 \\
\hline 72 & 2013-07-30 18 & 2013-08-02 18 & 3.25 & 2608 & -1.07 & 0.16 & 0.62 \\
\hline 73 & 2015-07-02 18 & 2015-07-09 18 & 7.25 & 2605 & 0.43 & 0.27 & 0.79 \\
\hline 74 & 2016-06-10 18 & 2016-06-14 18 & 4.25 & 2652 & -1.47 & 0.18 & 0.66 \\
\hline 75 & $2016-06-2218$ & $2016-06-2518$ & 3.25 & 2581 & 2.50 & 0.23 & 0.55 \\
\hline 76 & 2016-07-18 18 & 2016-07-24 18 & 6.25 & 2607 & 0.25 & 0.25 & 0.76 \\
\hline 77 & $2017-07-2512$ & $2017-07-2718$ & 2.5 & 2628 & 1.98 & 0.28 & 0.76 \\
\hline
\end{tabular}



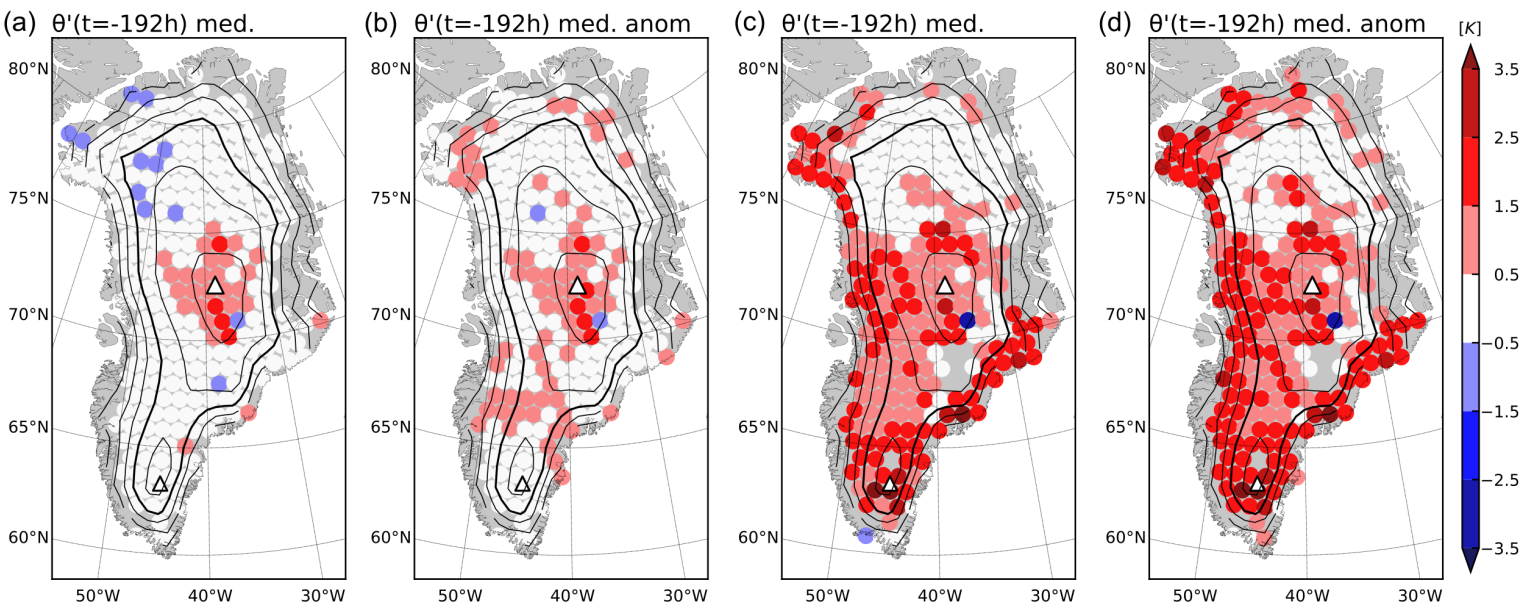

Figure S1. LFP maps of the median potential temperature anomaly $\theta^{\prime}$ wrt. local climatology $\theta_{c l}$ at $t=-192 \mathrm{~h}$ (a) during all melt events, (c) during EV69, and (b,d) the respective anomaly wrt. the climatological summertime air streams. The contours indicate elevation in $500 \mathrm{~m}$ intervals with the $2000 \mathrm{~m}$ isoline in solid. Summit and Southdome are marked with triangles. 

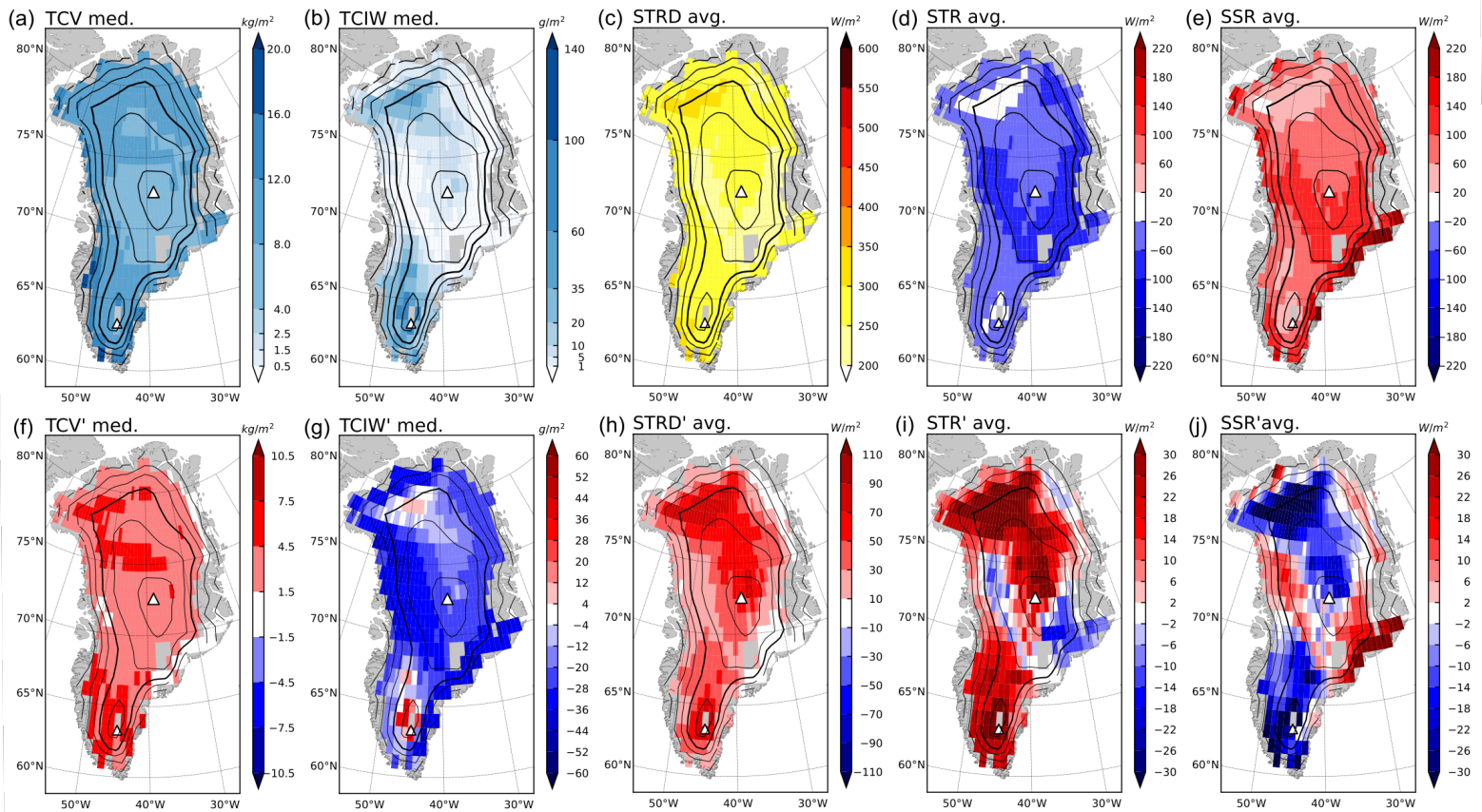

(g) TCIW' med
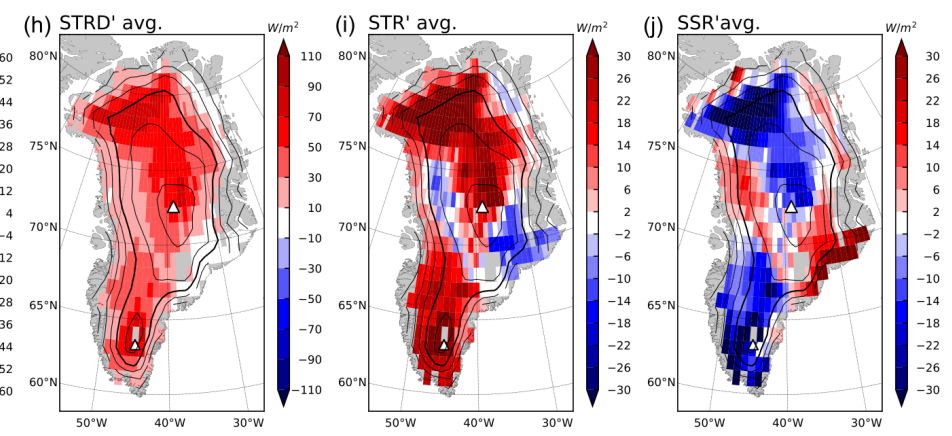

Figure S2. As Fig. 7 but for (a) total column water vapor (TCV), (b) total column liquid ice water (TCIW), (c) surface longwave downward radiation $(S T R D),(\mathrm{d})$ net surface longwave radiation $(S T R)$, (e) net surface shortwave radiation (SSR) during EV69 melt time steps, and (f-j) their anomalies wrt. climatology. 

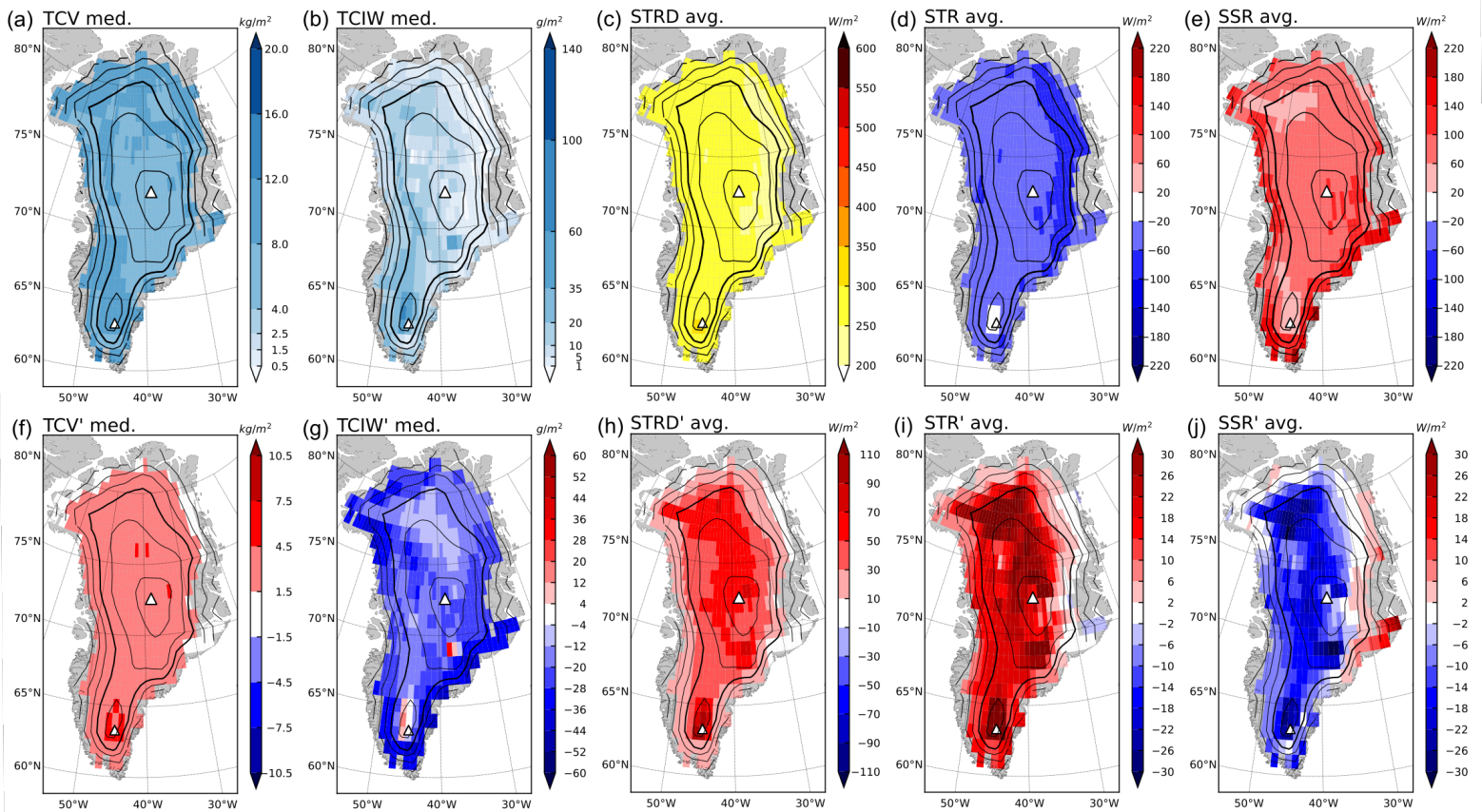

Figure S3. As Fig. S2 but for melt time steps during all Greenland melt events. 